Nota de pesquisa/ Research note

\title{
TRES CITAS NUEVAS DEL GÉNERO Ischnoptera BURMEISTER, 1838 (BLATTELLINAE, BLATTELLIDAE, BLATTARIA) PARA URUGUAY
}

\author{
Alejandra del C. Valverde \& Francisco A. Crespo
}

\begin{abstract}
Three new records for the genus Ischnoptera Burmeister, 1838 (Blattellinae, Blattellidae, Blattaria) from Uruguay - The number of species of the genus Ischnoptera reported from Uruguay is doubled, increasing its number to 6 . Ischnoptera argentina Hebard, 1921; I. bilunata Saussure, 1869; and I. litostylata Hebard, 1921 are reported for the first time. The available information on the species distribution is mapped.
\end{abstract}

Key words: Blattaria, Ischnoptera, cockroaches, distribution

El género Ischnoptera está incluido en la subfamilia Blattellinae, familia Blattellidae (Grandcolas, 1996). En Argentina es uno de los géneros mejor representados (10 spp), pero sólo cinco de ellas han sido halladas en las provincias limítrofes con Uruguay (I. argentina Hebard, 1921; I. bilunata Saussure, 1869; I. carcarana Hebard, 1921; I. ignobilis, Saussure, 1864 e I. litostylata Hebard, 1921).

Facultad de Ciencias Exactas y Naturales. UBA, Departamento de Biodiversidad y Biología Experimental, 4ํㅜ piso, Pabellón II, Ciudad Universitaria, C 1428 EHA Buenos Aires, Argentina. Fax: 054011 4576-3384. E-mail: valverde@bg.fcen.uba.ar

Recebido em: 20/07/2004.

Aceito em: 13/01/2005. 
Argentina y Uruguay tienen en común algunas regiones biogeográficas (Cabrera \& Willink, 1980), por lo tanto es de esperar que ambos países compartan la fauna de Blattaria (Crespo \& Valverde 2003). Según dicho razonamiento, se anticipaba la presencia de tres especies de Ischnoptera en la Provincia Biogeográfica Pampeana: I. argentina, I. bilunata e I. litostylata, elevando a 6 el número total de especies para el género Ischnoptera en la República Oriental del Uruguay (Fig. 1). Con el nuevo material legado a esta cátedra se confirma la presencia de estas especies en las localidades de Paso del Horno, Departamento de Canelones y Nueva Palmira, Departamento de Colonia, Uruguay.
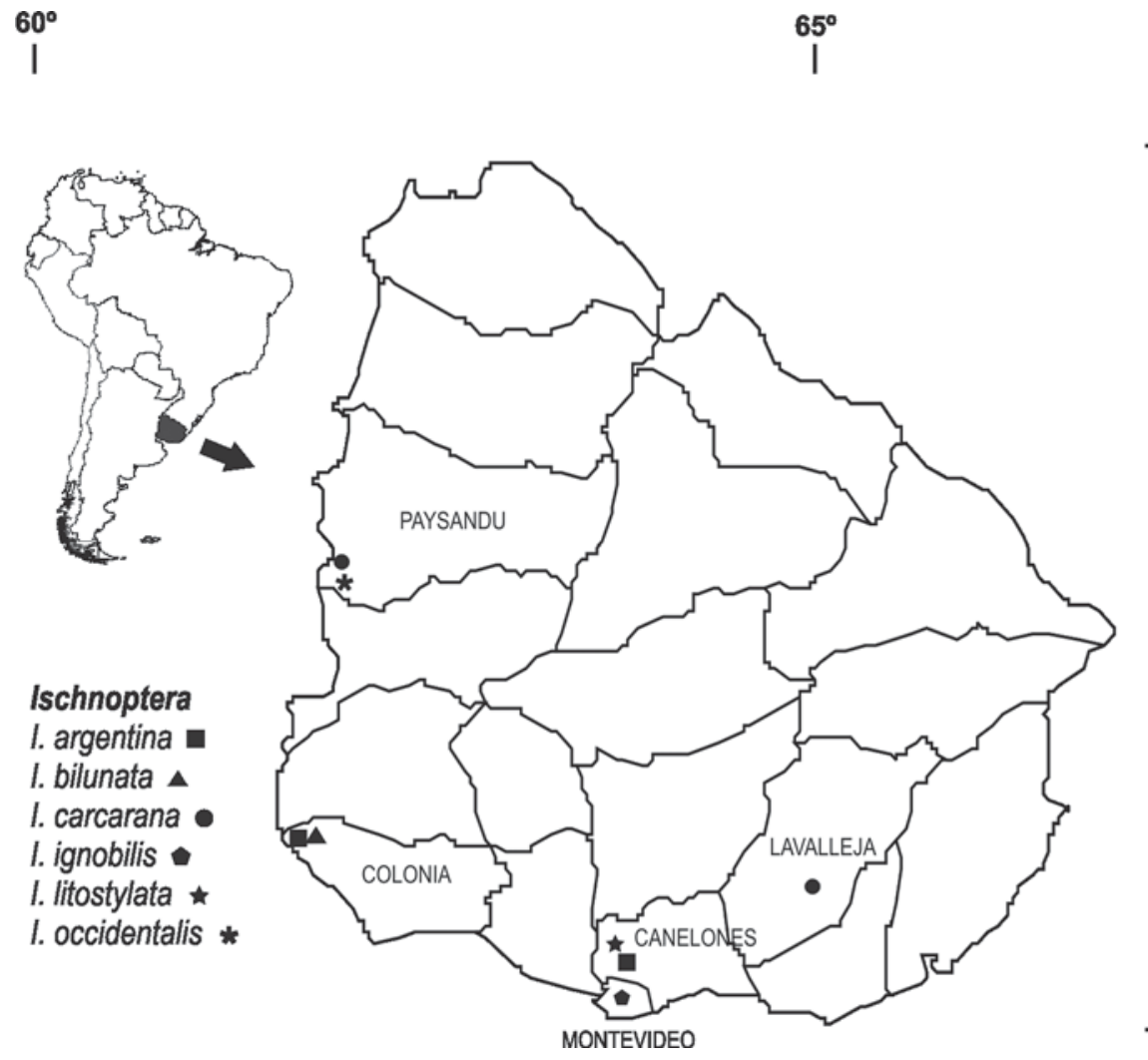

Figura 1: Distribución geográfica de Ischnoptera en Uruguay. 
Las siguientes especies ya fueron citadas para la República Oriental del Uruguay: I. carcarana Hebard, 1921 para los departamentos Paysandú y Lavalleja por Crespo y Valverde, 2003; I. ignobilis Saussure, 1864 para Montevideo por Hebard 1921 e I. occidentalis Saussure, 1862 para Paysandú por Bolívar 1884.

Material examinado: Las siguientes especies fueron determinadas mediante las descripciones de Hebard 1921.

I. argentina Hebard, 1921. URUGUAY, Canelones, paraje "Paso del Horno",

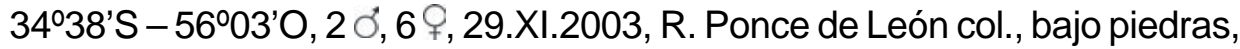
Facultad de Ciencias Exactas y Naturales (UBA); Colonia, Nueva Palmira,

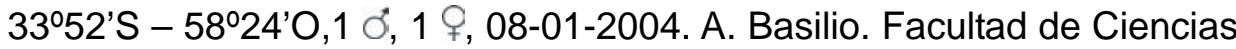
Exactas y Naturales (UBA).

I. bilunata Saussure, 1869. URUGUAY, Colonia, Nueva Palmira, 3352'S 5824'O, 4 Ó, 5 \&, 08-01-2004. A. Basilio. Facultad de Ciencias Exactas y Naturales (UBA).

I. litostylata Hebard, 1921. URUGUAY, Canelones, paraje "Paso del Horno",

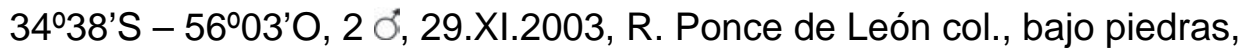
Facultad de Ciencias Exactas y Naturales (UBA).

\section{Agradecimientos}

Agradecemos al doctor Rodrigo Ponce de León de la Facultad de Ciencias de Montevideo y a la doctora Alicia Basilio de la Facultad de Ciencias Exactas y Naturales de Buenos Aires, quienes desinteresadamente nos enviaron el material colectado.

\section{Referencias Bibliográficas}

Cabrera, A.L. \& Willink, A., 1980. Biogeografía de América Latina. (Chesneau, E. V. Ed.). Editora OEA, Washington, D.C., 117 p. 
Crespo, F.A. \& Valverde, A.C., 2003. Introducción al conocimiento de las "cucarachas" del Uruguay (Blattaria). Entomol. Vect. 10:587-594.

Grandcolas, P., 1996. The phylogeny of cockroach families: a cladistic appraisal of morpho-anatomical data. Can. J. Zool. 74:508-527.

Hebard, M., 1921. South American Blattidae from the Museum D'Histoire Naturelle, Paris, France. Proc. Acad. Nat. Sci. Phil. 73:193-304. 